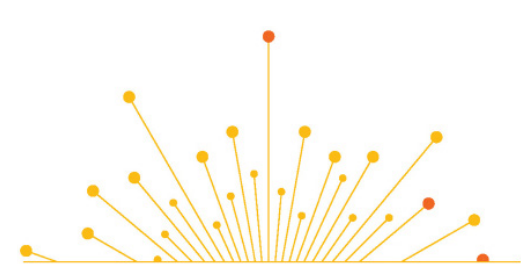

SCIENCE TABLE

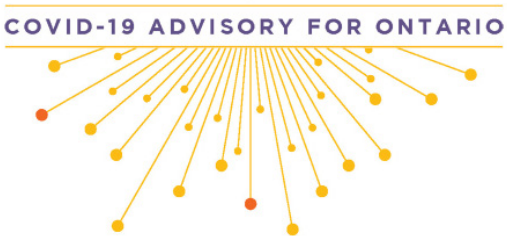

Version: $\mathbf{1 . 0}$

Published: September 14, 2021

Citation: Razak F, Katz GM, Cheung AM, et al. Understanding the post COVID-19 condition (long COVID) and the expected burden for Ontario. Science Briefs of the Ontario COVID-19 Science Advisory Table. 2021;2(44). https://doi.org/10.47326/ ocsat.2021.02.44.1.0

Author Affiliations: The affiliations of the members of the Ontario COVID-19 Science Advisory Table can be found at https:// covid19-sciencetable.ca/.

Declarations of Interest: The declarations of interest of the members of the Ontario COVID-19 Science Advisory Table, its Working Groups, or its partners can be found at https:// covid19-sciencetable.ca/. The declarations of interest of external authors can be found under additional resources at https://doi. org/10.47326/ocsat.2021.02.44.1.0

About Us: The Ontario COVID-19 Science Advisory Table is a group of scientific experts and health system leaders who evaluate and report on emerging evidence relevant to the COVID-19 pandemic, to inform Ontario's response. Our mandate is to provide weekly summaries of relevant scientific evidence for the COVID-19 Health Coordination Table of the Province of Ontario, integrating information from existing scientific tables, Ontario's universities and agencies, and the best global evidence. The Science Table summarizes its findings for the Health Coordination Table and the public in Science Briefs.

Correspondence to: Secretariat of the Ontario COVID-19 Science Advisory Table (info@covid19-sciencetable.ca)

Copyright: 2021 Ontario COVID-19 Science Advisory Table. This is an open access document distributed under the terms of the Creative Commons Attribution License, which permits unrestricted use, distribution, and reproduction in any medium, provided that the original work is properly cited.

The views and findings expressed in this Science Brief are those of the authors and do not necessarily reflect the views of all of the members of the Ontario COVID-19 Science Advisory Table, its Working Groups, and its

\title{
Understanding the Post COVID-19 Condition (Long COVID) and the Expected Burden for Ontario
}

Fahad Razak, Gabrielle M. Katz, Angela M. Cheung, Margaret S. Herridge, Arthur S. Slutsky, Upton Allen, Adalsteinn D. Brown, Gerald A. Evans, David M. Kaplan, Linda Mah, Laveena Munshi, Douglas G. Manuel, Andrew M. Morris, Paula A. Rochon, Kieran L. Quinn, Beate Sander, Brian Schwartz, Arjumand Siddiqi, Ashleigh Tuite, Amol A. Verma, Peter Jüni, Karen B. Born on behalf of the Ontario COVID-19 Science Advisory Table

\section{Key Message}

The "post COVID-19 condition" (or long COVID) describes a range of symptoms which can persist for months after severe, mildly symptomatic or asymptomatic SARS-CoV-2 infection. The most common of more than 200 reported symptoms include fatigue, shortness of breath, pain, sleep disturbances, anxiety, and depression.

Many people with the post COVID-19 condition have difficulty returning to baseline levels of function and have high rates of health care utilization. A conservative estimate suggests that 57,000 to 78,000 Ontarians had or are currently experiencing the post COVID-19 condition, although prevalence estimates can vary widely depending on the case-definition applied. Vaccination is likely protective against development of the post COVID-19 condition.

More research is required to develop a consensus definition of the post COVID-19 condition, understand risk factors including the role of viral variants, quantify the impact on specific populations such as children, and develop strategies for prevention and treatment.

\section{Summary}

\section{Background}

The term "post COVID-19 condition" encompasses numerous potential sequelae and symptoms that may persist for weeks to months following infection with SARSCoV-2. In some individuals, these symptoms can lead to impairment of home and work life, and increased use of healthcare services. A comprehensive understanding of the post COVID-19 condition may aid decision-makers and health care systems in developing and implementing future policies and health resource planning related to this condition.

\section{Questions}

How has the post COVID-19 condition been defined?

What are the potential underlying causes of the post COVID-19 condition?

What is the prevalence of the post COVID-19 condition and its associated symptoms and sequelae?

Who is at risk of developing the post COVID-19 condition, and can this risk be mitigated?

How does the post COVID-19 condition affect patterns of health system utilization, functional limitations, and utilization of long-term disability and unemployment 
benefits?

What is the expected burden of the post COVID-19 condition for Ontario?

\section{Findings}

More than 200 different symptoms across 10 organ systems have been associated with the post COVID-19 condition, and some of the most prevalent symptoms include fatigue, shortness of breath, general pain or discomfort, anxiety, and depression. The post COVID-19 condition can develop among those who had severe, mildly symptomatic, or asymptomatic infection. The World Health Organization (WHO) has reported that approximately 1 in 4 individuals who were infected with SARS-CoV-2 experience symptoms of post COVID-19 condition for at least 1 month, and 1 in 10 experience symptoms lasting beyond 12 weeks. However, prevalence estimates vary widely depending on factors such as the case definition, sampling strategy, severity of initial COVID-19 infection, or time interval before follow-up. Patients with the most severe illness during initial infection, and especially those who require intensive care unit (ICU) admission, are expected to have significant long-term health consequences.

Risk factors for the post COVID-19 condition require further study. Based on a WHO brief, potential risk factors include higher body mass index (BMI), being female, being older, and having more acute symptoms or having more severe COVID-19 infection. Vaccination against COVID-19 reduces the likelihood of the post COVID-19 condition by reducing the chance of becoming infected. In addition, vaccination may reduce the likelihood of developing the post COVID-19 condition among those with post vaccine ("breakthrough") infections.

Individuals with the post COVID-19 condition may have impaired cognitive and physical functional status, including limitations in the ability to perform activities of daily living (ADL); tasks such as dressing or bathing that are necessary to independently care for oneself), reduced ability to care for family members or dependents, difficulty returning to work and increased health care use. A conservative estimate suggests that between 57,000 to 78,000 individuals had or are currently experiencing the post COVID-19 condition in Ontario.

\section{Interpretation}

The post COVID-19 condition is associated with a wide range of ongoing symptoms and morbidity, increased use of health care resources, and adverse impact on work and activities of daily living. The post COVID-19 condition has substantial potential harms for patients, caregivers, health systems and society.

Estimates of the prevalence and burden of the post COVID-19 condition depend on the specific definition applied, which is evolving. However, conservative estimates drawing from a variety of definitions suggest 57,000 to 78,000 Ontarians had or might be presently affected by the post-COVID 19 condition. Health system management and supportive policies are needed for Ontario to manage the burden of post COVID-19 condition.

\section{Background}

As of September 3, 2021 there have been more than 567,000 cases of SARS-CoV-2 infection in Ontario. ${ }^{1}$ Although the majority of individuals with COVID-19 fully recover, some may not return to their pre-illness levels of health even weeks to months after infection. The persistence of symptoms post infection were noted as early as the summer of 2020 in both the academic literature ${ }^{2}$ in the press and in social media. ${ }^{3}$

The post COVID-19 condition, ${ }^{4}$ also known as "Long COVID", is a term that encompasses numerous potential sequelae of infection with SARS-CoV-2 that may vary across 
factors such as the severity of illness, vaccination status, and the intensity of care required. The pathophysiology underlying the post COVID-19 condition may include the direct effects of infection and factors related to any severe illness that requires hospitalization and/or critical care admission.

Defining the post COVID-19 condition remains a central challenge in the literature and impairs unified estimates of critical features such as prevalence and outcome measures. This brief examines a broad array of the current literature, including preprints. A comprehensive understanding of the post COVID-19 condition may aid in the clinical care of individual patients and the development and implementation of policy and health system management decisions.

\section{Questions}

How has the post COVID-19 condition been defined?

What are the potential underlying causes of the post COVID-19 condition?

What is the prevalence of the post COVID-19 condition and its associated symptoms and sequelae?

Who is at risk of developing the post COVID-19 condition, and can this risk be mitigated?

How does the post COVID-19 condition affect patterns of health system utilization, functional limitations, and utilization of long-term disability and unemployment benefits?

What is the expected burden of the post COVID-19 condition for Ontario?

\section{Findings}

\section{Defining the Post COVID-19 Condition}

Definitions are critical to understanding the epidemiology and true burden of the symptoms and sequelae that constitute the post COVID-19 condition. However, there is a lack of consensus on definitions and terminology (Table 1), and consequently, this brief draws on a global, heterogeneous literature which uses varying definitions of the post COVID-19 condition to provide a comprehensive overview of this condition.

\begin{tabular}{|c|c|c|}
\hline Origin & Term & Definition \\
\hline \multirow[t]{3}{*}{$\begin{array}{l}\text { National Institute for } \\
\text { Clinical Excellence (NICE) }\end{array}$} & $\begin{array}{l}\text { Long COVID (includes } \\
\text { ongoing symptomat- } \\
\text { ic COVID-19 and post- } \\
\text { COVID-19 syndrome) }\end{array}$ & $\begin{array}{l}\text { Long COVID is characterized as the persistence of } \\
\text { COVID- } 19 \text { signs and symptoms that continue to develop } \\
\text { after acute COVID- } 19 \text { and can include both ongoing } \\
\text { symptomatic COVID- } 19 \text { and post-COVID- } 19 \text { syndrome. }\end{array}$ \\
\hline & & $\begin{array}{l}\text { Ongoing symptomatic COVID-19 } \\
\text { Signs and symptoms which persist from } 4 \text { to } 12 \text { weeks. }\end{array}$ \\
\hline & & $\begin{array}{l}\text { Post-COVID-19 syndrome } \\
\text { Signs and symptoms that develop during or after an } \\
\text { infection consistent with COVID-19, continue for more } \\
\text { than } 12 \text { weeks and are not explained by an alternate } \\
\text { diagnosis. }\end{array}$ \\
\hline $\begin{array}{l}\text { Centers for Disease } \\
\text { Control and Prevention } \\
(C D C)^{6}\end{array}$ & Post-COVID conditions & $\begin{array}{l}\text { New, returning, or ongoing health problems that people } \\
\text { experience } 4 \text { or more weeks after initial infection with } \\
\text { SARS-CoV-2. }\end{array}$ \\
\hline $\begin{array}{l}\text { World Health Organization } \\
(\mathrm{WHO})^{4}\end{array}$ & Post COVID-19 condition & $\begin{array}{l}\text { Post COVID-19 condition can be assumed in a patient } \\
\text { who does not recover to baseline health after an initial } \\
\text { infection or diagnosis of COVID-19. }\end{array}$ \\
\hline $\begin{array}{l}\text { Public Health Agency of } \\
\text { Canada }(\mathrm{PHAC})^{7}\end{array}$ & Post COVID-19 conditions & $\begin{array}{l}\text { Symptoms persisting or recurring for weeks after acute } \\
\text { COVID-19 illness. } \\
\text { This can be further broken down into post COVID-19 } \\
\text { conditions occurring } 4-12 \text { weeks (short term) and }>12 \\
\text { weeks (long-term) after COVID-19 diagnosis }\end{array}$ \\
\hline
\end{tabular}


Table 1. Terminology and Definitions Describing Post COVID-19 Condition

Table presenting the current definitions and terminology used to describe the post COVID-19 condition in the literature.

\section{The Post COVID-19 Condition: A Disease Framework and Potential Underlying Causes}

A WHO report on the post COVID-19 condition includes two overlapping disease constructs (Figure 1). The first represents individuals with prolonged symptoms that are related to the infection itself and can include individuals who were initially asymptomatic, non-hospitalized, or not even tested for SARS-CoV-2. The symptoms are wide ranging and can wax and wane. The most reported symptoms include fatigue, dyspnea, pain, weakness, sleep disturbances, and cognitive dysfunction (e.g., "brain fog"), anxiety and depression. The second construct represents symptoms and sequelae commonly observed in survivors of critical illness and hospitalization, ${ }^{4}$ especially among those who are admitted to the ICU. The Post-intensive care syndrome (PICS) is a well-documented condition of acquired disability after critical illness leading to impairment of cognition, psychological health and physical function. ${ }^{8}$

Post COVID-19 Condition
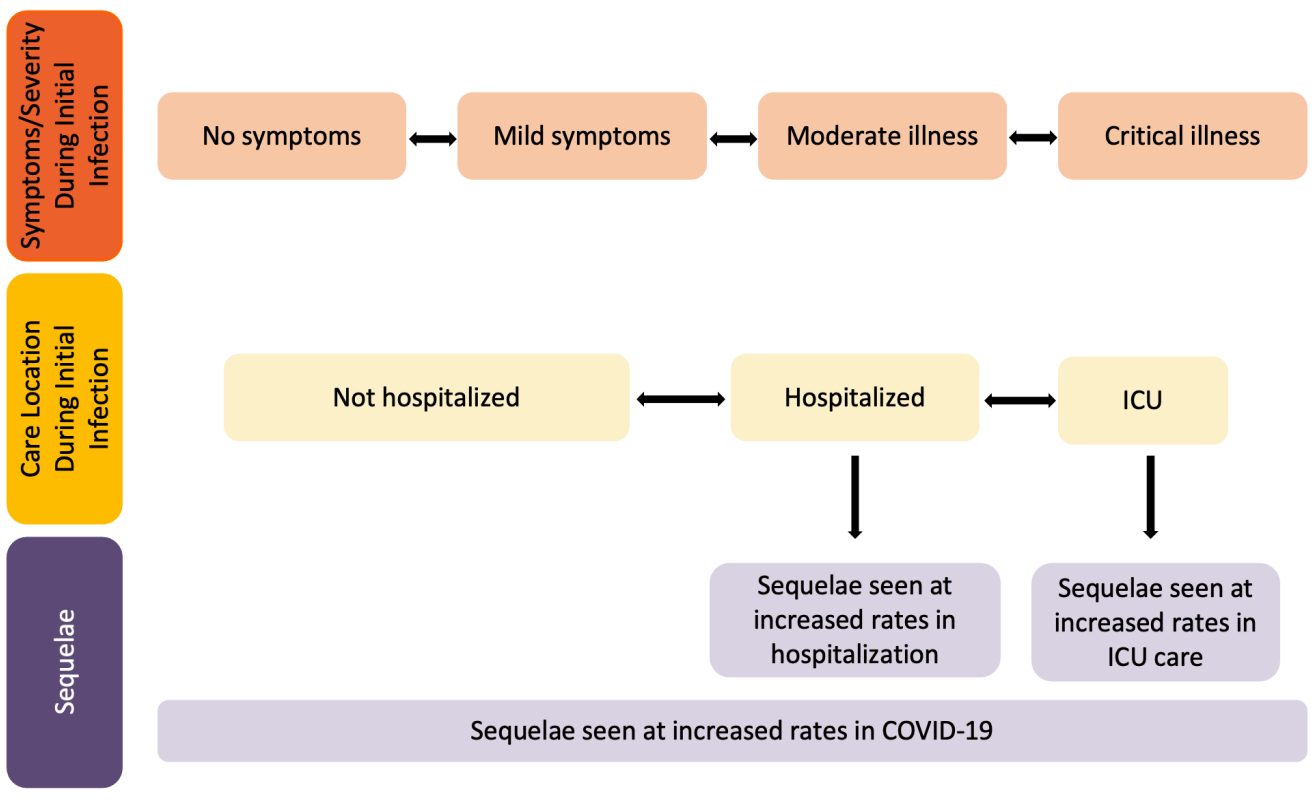

Figure 1. General Overview of Post COVID-19 Condition

Figure presenting a high-level overview of the post COVID-19 condition according to symptoms and severity, care location, and sequelae. Note that symptoms and severity of initial SARS-CoV-2 and care location may not be the sole determinants of sequelae; for example, age and length of stay in ICU may influence the observed sequelae in post ICU patients. ${ }^{9} \mathrm{ICU}$, intensive care unit. Figure adapted from the World Health Organization. ${ }^{4}$

The SARS-CoV-2 virus enters the cell via the angiotensin converting enzyme 2 (ACE2) receptor and then undergoes replication. More than 200 different symptoms across 10 organ systems have been associated with the post COVID-19 condition, ${ }^{10}$ including nearly every organ or physiologic system: ${ }^{11}$ central nervous (e.g., brain fog, fatigue), musculoskeletal (e.g., weakness, joint pain), respiratory (e.g., dyspnea), gastrointestinal (e.g., diarrhea), cardiac (e.g., arrhythmias), circulatory (e.g., vasculitis), and psychological (e.g., mood swings, insomnia) systems (Figure 2). ${ }^{6,11-13}$

How SARS-CoV-2 infection leads to the post COVID-19 condition is an area of intense study. There is growing evidence that the virus can cause organ damage (e.g., lung scaring) and give rise to physiologic dysfunction such as increased blood clotting, vasculitis or proinflammatory response. A National Institute of Health Research (NIHR, United Kingdom (UK)) review summarized multiple potential contributing mechanisms driving the constellation of symptoms and findings in the post COVID-19 condition: ${ }^{14}$ 


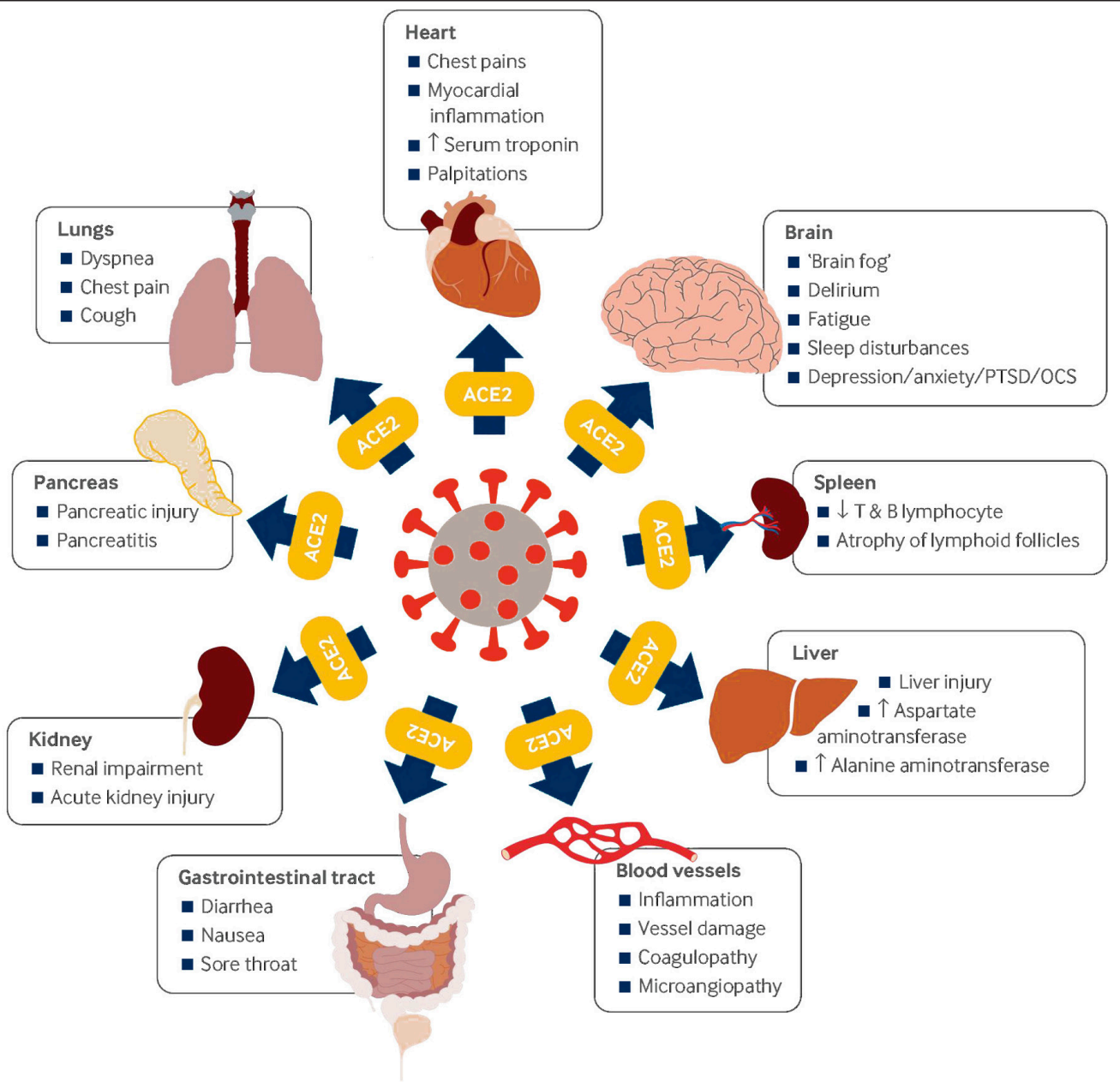

Figure 2. Impact of COVID-19 and Post-COVID Condition on Various Organ Systems

PTSD, post-traumatic stress disorder. OCS, obsessive-compulsive symptoms. Reproduction with permission from the BMJ (Crook et al, 2021). ${ }^{11}$

\section{Neurologic Dysfunction}

A review of the neurologic impact of COVID-19 identified two major categories: 1) acute and rarely occurring severe consequences such as Guillain-Barré syndrome or encephalitis, and 2) chronic, highly variable, and often time-varying symptoms which overlap with conditions such as myalgic encephalomyelitis/chronic fatigue syndrome (ME/CFS). ${ }^{15}$ Symptoms such as dyspnea, myalgia (muscle pain), exercise intolerance and sleep disturbances that are commonly reported in the post COVID-19 condition have some similarities to symptom complexes noted in ME/CFS. ${ }^{15}$

Fatigue is one of the most commonly reported cognitive symptoms by individuals with the post COVID-19 condition, and was reported by $40 \%$ of individuals post infection with influenza or coronaviruses such as severe acute respiratory syndrome (SARS) and Middle East respiratory syndrome (MERS). ${ }^{16}$ Persistent post-viral fatigue beyond 6 months was noted post SARS infection in approximately one quarter of patients. ${ }^{17}$

\section{Immune Related Phenomena}

Medications such as dexamethasone and tocilizumab that modulate or suppress immune response have been effective short-term therapies in reducing the morbidity and mortality associated with severe COVID-19. ${ }^{18,19}$ The pathophysiology of the post COVID-19 condition also likely has a significant underlying immune component, and the British Society for Immunology has summarized a broad range of relevant immune factors, including: excess immune response to the virus, post-viral autoimmunity, persistent inflammation and thrombotic complications. ${ }^{20}$ 
For example, a study on COVID-19 patients and matched controls followed for 2-3 months post infection demonstrated that markers of immune activation (C-reactive protein (CRP) and white blood cell count) correlated with degree of damage across a range of organ systems: cardiopulmonary exercise test parameters, white matter hyperintensity volume on brain magnetic resonance imagining (MRI), and MRI measures of fibrosis in the heart, liver and kidney. ${ }^{21}$

\section{Organ Dysfunction}

Persistent organ damage and reduced organ function may partially contribute to many of the symptoms and findings in the post COVID-19 condition. A recent review examined factors that can lead to persistent organ dysfunction, including damage during acute infection causing fibrosis and scarring, sustained production of inflammatory cytokines, and endothelial damage and vascular abnormalities. ${ }^{11}$ These mechanisms seem to be implicated in clinical findings noted to occur in higher frequency across major organ systems such as the lung, heart and central nervous system, in patients with the post COVID-19 condition. For example, changes in lung function can be driven by weakness in muscles of respiration, chronic inflammation and endothelial damage. This can lead to reported symptoms and sequelae such as dyspnea, abnormalities in pulmonary function testing, ${ }^{22}$ persistent damage on lung imaging studies, ${ }^{23}$ and increased risk of thrombosis. ${ }^{24}$

In the heart, chronic inflammation leads to fibrosis and scarring, resulting in damage to cardiomyocytes and to the afferent autonomic nervous system. These underlying abnormalities may drive many of the reported cardiac symptoms in the post COVID-19 condition including chest pain and arrhythmias. ${ }^{2}$ Troponin elevation can exist more than 2 months post infection suggesting ongoing cellular damage in the post-acute phase. ${ }^{25}$

Long-term kidney dysfunction is also linked to the post COVID-19 condition. A Veterans Health (United States) study of 89,216 individuals with COVID-19 and 1,637,467 noninfected controls compared kidney outcomes for individuals who survived for at least 30 days following a positive SARS-CoV-2 test. ${ }^{26}$ At 30 days, individuals with COVID-19 exhibited a higher risk of acute kidney injury, decreased renal function, and a higher likelihood of end stage kidney disease and major adverse kidney events. Further, the study found that individuals with COVID-19 who had more severe illness and hospitalization were more likely to have worse kidney outcomes than those who were not hospitalized or had milder illness.

\section{Control Comparisons}

Few studies examining the post COVID-19 condition have used comparative controls, ${ }^{14}$ and this is a crucial area for future research given the need to disentangle disease-related effects from those related to processes of care or other factors. Existing studies using controls demonstrate that certain enduring symptoms are more common following SARS-CoV-2 infection. For example, a study used routinely recorded health data from hospitals in England to compare 47,480 individuals hospitalized with COVID-19 to matched non-hospitalized controls not positive for COVID-19 from the UK's general population. ${ }^{27}$ Each control was matched on an extensive set of criteria including personal and demographic factors (e.g., age, sex, ethnicity, etc.), comorbidities, smoking status and body mass index. Rehospitalization and mortality within 140 days were 3.5 and 7.7 times more likely, respectively, in individuals hospitalized with COVID-19 compared to controls. Additionally, incidence of respiratory disease, diabetes, and major cardiovascular events were 6 times, 1.5 times, and 3 times higher, respectively, in patients with COVID-19 compared to controls. However, it is difficult to disentangle the effects of COVID-19 from hospitalization or other factors in this retrospective cohort study. 
Astudy examining 58 hospitalized COVID-19 patients and 30 age, sex, BMI, comorbiditymatched hospitalized controls admitted for non-COVID-19 reasons. ${ }^{21}$ Notably, approximately one third of the hospitalized COVID-19 patients were admitted to ICU, yet none of the hospitalized controls were. At follow-up 2-3 months post discharge, patients hospitalized with COVID-19 had increased abnormalities on MRI of heart and liver, and reduced exercise intolerance, and these abnormalities correlated with serum markers of inflammation (e.g., C-reactive protein) and acute illness severity during hospitalization.

A study of cross-sectional cognitive performance data from 81,337 participants from the UK included individuals who self-identified a previously having COVID-19. ${ }^{28}$ COVID-19 infection status was only asked about after cognitive testing was completed. Compared to controls matched for age, gender, education level, income, racial-ethnic group, and pre-existing medical disorders, individuals who self-identified as having suspected COVID-19 or reported a positive COVID-19 test had significantly lower cognitive performance.

\section{Prevalence of Post COVID-19 Condition and Associated Symptoms and Sequelae}

Given differing definitions of the post COVID-19 condition, it is not possible to give precise prevalence estimates. Important factors that will impact prevalence estimates across studies include: 1 ) severity of initial infection (ranging from asymptomatic to critical illness), 2) time interval used to assess outcomes and symptoms/sequelae that are assessed, 3) bias in patient recruitment, including sampling strategy (e.g., population-based versus self-report via an app) and criteria for enrolment (e.g., positive COVID-19 test versus plausible symptoms with an epidemiologic-link), 4) sample size of each study, 5) health system capacity for SARS-CoV-2 testing, 6) the episodic nature of symptoms for some individuals, and 7) the impact of emerging therapeutics and viral variants. Given these challenges, the reported prevalence of post COVID-19 condition and its associated symptoms and sequelae greatly varies across jurisdictions, from $2 \%{ }^{29}$ to $89 \%{ }^{30}$ in one NIHR Review. ${ }^{14}$

The WHO has reported that approximately 1 in 4 individuals who were infected with SARS-CoV-2 experience symptoms of post COVID-19 condition for at least 1 month, and 1 in 10 experience symptoms lasting beyond 12 weeks. ${ }^{31}$ The UK Office for National Statistics April 2021 report examined a population-representative sample of individuals in the UK. $21 \%$ of respondents reported symptoms at 5 weeks post SARS-CoV-2 infection; $13.7 \%$ of respondents reported symptoms at 12 weeks post infection. ${ }^{32}$ Initial findings from a living systematic review coordinated by the Public Health Agency of Canada (PHAC) on the post COVID-19 condition indicate that most patients with laboratory-confirmed COVID-19 continued to experience 1 or more of over 100 symptoms within 4-12 weeks (83\%) and beyond 12 weeks (56\%) after diagnosis, regardless of illness severity. ${ }^{7}$ However, given that certain symptoms (e.g., headache, fatigue) are also common and episodic in individuals who have not had COVID-19 and lack of non-COVID-19 controls, the PHAC estimates may be high.

The pattern and prevalence of symptoms change over time, and a common framework describes short-term (4-12 weeks after COVID-19 diagnosis) and long-term ( $>12$ weeks after COVID-19 diagnosis) sequelae associated with post COVID-19 condition. ${ }^{7}$ The PHAC systematic review reports the frequencies of the most prevalent short-term and long-term symptoms and sequelae (Table 2). 


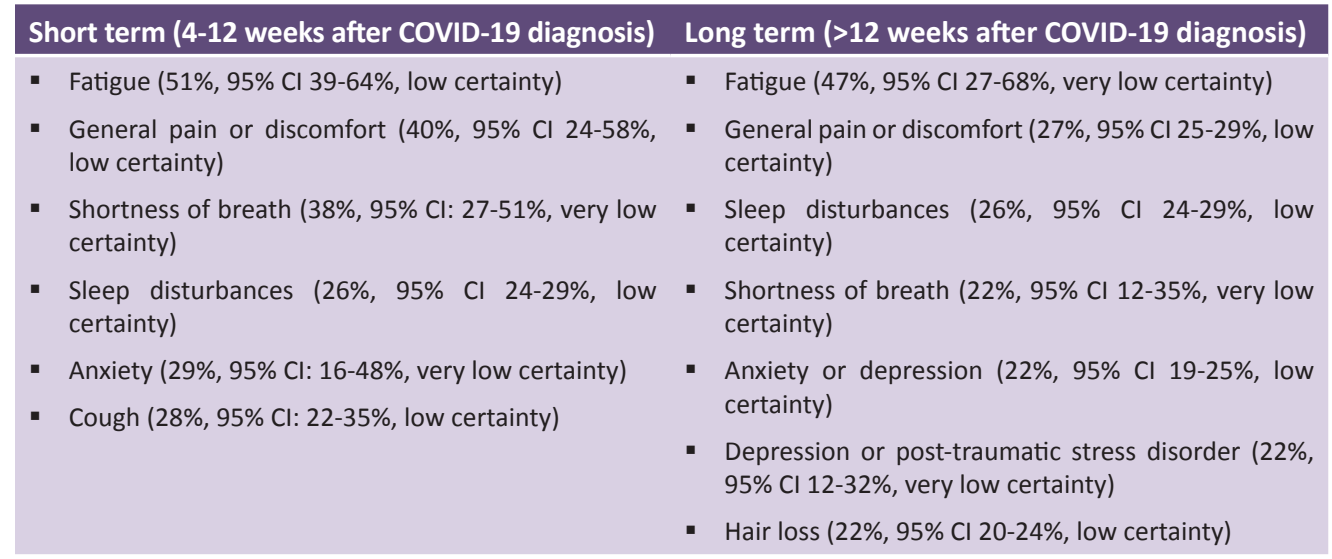

Table 2. Prevalence of Symptoms and Sequelae Associated with Post COVID-19 Condition

Table presenting the symptoms and sequelae associated with the post COVID-19 condition that are most prevalent in the short term (4-12 weeks following COVID-19 diagnosis) and the long term (>12 weeks following COVID-19 diagnosis). Each symptom is listed followed by its reported prevalence, 95\% confidence interval (CI), and GRADE assessment (which gives a sense of how much value can be placed on each result given data limitations). Data sourced from Reyes Domingo et al. ${ }^{7}$

\section{Individuals Previously Hospitalized Due to COVID-19}

Individuals with severe COVID-19 infection may be more likely to develop the post COVID-19 condition but disentangling the impact of COVID-19 infection from processes of critical illness care (e.g., severe of lung injury in an individual on mechanical ventilation) is challenging. A study conducted in Wuhan, China $(1,733$ patients previously hospitalized due to COVID-19; 76 patients in the ICU) examining the longterm consequences of COVID-19 after hospital discharge found that $76 \%$ reported new and persistent symptoms after 6 months. ${ }^{33}$ Patients most commonly reported experiencing at least one of the following symptoms of fatigue, muscle weakness, sleep difficulties, anxiety or depression. Those with more severe illness during their initial infection were found at follow-up to have greater impairment of pulmonary diffusion capacity and increased rates of chest imaging anomalies on CT scan (e.g., volume of lung consolidation), but this finding has also seen more broadly in the acute respiratory distress syndrome (ARDS) literature in non-COVID-19 patients as well.

A study from the United Kingdom analyzed the clinical outcomes for 47,780 individuals with COVID-19 who were discharged from hospital, compared to non-hospitalized controls not positive for SARS-CoV-2 matched on clinical and demographic features. ${ }^{27}$ Rates of hospital readmission and mortality were 3.5 and 7.7 times greater in the previously hospitalized group of COVID-19 patients, respectively. In addition, diagnoses for new onset respiratory disease, major cardiovascular events, and diabetes were 27.3, 3.0, and 1.5 times more frequent than in the control group. In this study, it is difficult to isolate the effects of COVID-19 illness from the impact of hospitalization as a decrement in health status is often seen following hospitalization for a wide range of conditions. Future studies using active controls (e.g., influenza or sepsis) may help to disentangle the effects of COVID-19 illness specifically.

\section{Duration of Symptoms}

A continuation of the Wuhan 6-month follow-up cohort study of hospitalized COVID-19 patients re-examined health status and other outcomes at 12 months. ${ }^{34}$ The proportion of patients with at least one sequelae or symptom decreased from $68 \%$ at 6 months to $49 \%$ at 12 months. Fatigue and muscle weakness were the two most reported symptoms during both follow-up visits, and the proportion fell from $52 \%$ at 6 months to $20 \%$ at 12 months. The proportion of patients with anxiety or depression at 12 months was slightly higher (23\% at 6-month visit; $26 \%$ at 12 -month visit).

At 12-month follow-up, health status (measured by sequelae or symptoms, lung 
diffusion capacity impairment, and radiographic abnormalities) was lower among COVID-19 survivors than in controls with no history of lab-confirmed SARS-CoV-2 infection (matched for age, sex, and comorbidities). 12 months after infection, 12\% had not returned to work, and of the $88 \%$ who had returned, $24 \%$ had not returned to their pre-COVID-19 level of work.

\section{Risk Factors for the Post COVID-19 Condition}

There is limited evidence that symptoms, clinical findings, or baseline risk can accurately predict who will develop the post COVID-19 condition. Several risk factors for developing the post COVID-19 condition following recovery from acute COVID-19 have been identified by a WHO rapid evidence review and individual studies: ${ }^{31}$

- Having a higher acuity of SARS-CoV-2 infection or presence of many acute COVID-19 symptoms s, $36^{36}$

- Having a higher BMI (obesity) ${ }^{35}$

- Being female ${ }^{5,35,37}$

- Being of older age $35,38,39$

In general, there has been limited study of whether chronic psychiatric conditions are risk factors for the post COVID-19 condition, or whether certain socioeconomic groups are differentially affected. ${ }^{35,38,39}$

A predictive model for the post COVID-19 condition was developed using age, sex, and the number of symptoms during the first week of COVID-19 illness. The model separated short duration of COVID-19 symptoms ( $<10$ days) from the post COVID-19 condition ( $\geq 28$ days of symptoms) with an area under the receiver operating characteristic curve of $76 \%$ on a replication sample of 2,472 individuals. ${ }^{35}$ This model may have direct applications in clinical settings to identify higher-risk groups and determine who may benefit from early intervention or surveillance. For example, a highly sensitive cut-off could be used to select patients for further monitoring for the development of the post COVID-19 condition with a positive predictive value of $34 \%$ and negative predictive value of $98 \%$.

COVID-19 vaccination reduces the probability of developing the post COVID-19 condition by greatly reducing the chance of becoming infected once fully vaccinated. In addition, there is emerging evidence that COVID-vaccination reduces the risk of developing the post COVID-19 condition even in post-vaccine ("breakthrough") infections. In a prospective cohort of 1,497 Israeli health care workers, ${ }^{39}(2.6 \%)$ had confirmed post-vaccine SARS-CoV-2 infections, and 7 ( $0.4 \%$ of sample; $19 \%$ of postvaccine infection cases) had post COVID-19 symptoms at 6 weeks. ${ }^{40}$ These included a prolonged loss of smell, persistent cough, fatigue, weakness, dyspnea, or myalgia. A prospective, community-based case-control study examining post- vaccine infections ( 7 days after second dose) in the UK found that the odds ratio of post COVID-19 condition lasting at least 28 days was $0.51(95 \% \mathrm{Cl} 0.32-0.82)$ in individuals infected after vaccination compared to infected non-vaccinated controls. ${ }^{41}$

Despite the importance of considering sex and age in identifying risk factors for developing post-COVID-19 condition, sex and age disaggregated data are seldom reported. This makes it more difficult to see patterns emerge that may help our understanding of the biological and social circumstances that may contribute to the post COVID-19 condition. $^{42,43}$

\section{Post COVID-19 Condition in Children}

Systematic reviews of SARS-CoV-2 infection in children have described mild disease and generally good outcomes in this population. ${ }^{44}$ The prevalence and characteristics of the post COVID-19 condition in children however, is less understood. A prospective 
UK cohort study of children aged 5 to 17 with positive SARS-CoV-2 test results from September 1, 2021 to January 24, 2021 found that 4.4\% experienced prolonged symptoms 28 days after infection, and $1.8 \%$ experienced symptoms 56 days after infection. ${ }^{45}$ The most common prolonged symptoms reported were fatigue, headache, loss of smell, and sore throat.

These findings are in line with smaller cohort studies of post COVID-19 condition prevalence, symptoms and health care use after SARS-CoV-2 infection in children. A study of 109 Swiss children who tested positive for SARS-CoV-2 antibodies found that $4 \%$ reported symptoms lasting beyond 12 weeks of infection, the most common being tiredness and difficulty concentrating ${ }^{47} \mathrm{~A}$ preprint exploring health care use of 10,306 children in Norway who tested positive for SARS-CoV-2 found a substantial increase in primary care use for mainly respiratory complaints in the first month following a positive test, and then increased primary care use for up to 3 months. The study found no increase in specialist care use for children who tested positive for SARS-CoV-2. ${ }^{48}$ It is challenging to contextualize these findings as there is a lack of control data using other groups such as those who were not infected or those infected with other viral infections (e.g., respiratory syncytial virus (RSV) infections). Further, the study does not take into account the emergence of new variants, including the SARS-CoV-2 Delta variant (B.1.617.2), which is more severe and transmissible in children and adults than prior variants. ${ }^{46}$

\section{Patterns of Medical/Health System Utilization in the Post COVID-19 Condition}

There is limited Canadian data on health care utilization patterns (e.g., specialist visits, emergency department visits, hospital admissions, end of life care) for patients with the post COVID-19 condition. Such data are essential given limited generalizability of findings from other jurisdictions which may have different models of healthcare coverage, payment structures and care pathways and infrastructure. There is growing international literature on health care utilization patterns in the months following hospital discharge; ${ }^{33,47-49}$ however, less is known about the health care needs and utilization of patients with post COVID-19 condition who were not hospitalized. A large pan-Canadian study will be examining this question (https://www.cancov.net/).

\section{Hospitalized Patients}

Different time frames have been applied to examine health care utilization of patients discharged after COVID-19 hospitalization. Studies following patients hospitalized for COVID-19 in the United States (US) have found 60-day rehospitalization rates to range from $15.1 \%$ to $19.9 \%{ }^{50,51} \mathrm{~A}$ study of patients in an Illinois hospital network found readmission rates within 30 days and 4 months respectively of $12.4 \%$ and $21.5 \% .^{52} \mathrm{~A}$ retrospective cohort study of 47,780 hospitalized COVID-19 patients in England found that in the 140 days ( 4.5 months) following discharge, nearly $33 \%$ were readmitted, and $10 \%$ died. ${ }^{27}$ Rates of readmission and death were 4 and 8 times greater than matched non-hospitalized control group, respectively.

Another metric of health care use after COVID-19 hospitalization that has been studied in the United States is out-of-pocket health care spending for patients within 90 days of discharge..$^{53}$ For privately insured patients, post-discharge out-of-pocket spending was higher than those hospitalized for pneumonia. This is suggestive of substantial financial burden for post-discharge health care although findings may not extend to Canada's single-payer system.

\section{Non-Hospitalized Patients}

Re-hospitalization may only represent the more severe spectrum of healthcare utilization, with more recent studies of non-hospitalized patients showing increased clinic, urgent care, and other community-based health care provider visits in the 
months following COVID-19 diagnosis. Electronic health record data from Kaiser Permanente Georgia of 3,171 non-hospitalized adults with COVID-19 found that $69 \%$ had one or more outpatient visits 28 to 180 days after the diagnosis. ${ }^{54}$ Two thirds had a visit for a new primary diagnosis, and approximately one third had a new specialist visit. Symptoms potentially related to COVID-19 were common among new visit diagnosis codes. Visits for these symptoms decreased after 60 days for most patients but for some patients continued through 120 to 180 days.

\section{Functional Limitations Typical of Post COVID-19 Condition and Impact on Work and Non-Work Life}

Functional limitations in the post COVID-19 condition may be related to major symptoms and sequelae such as fatigue, dyspnea, weakness, and mood disturbances and these may be more severe in patients hospitalized at the time of initial infection. ${ }^{33}$

These symptoms and sequelae may limit an individual's ability to perform ADLs which are defined as those activities required to independently care for oneself. ${ }^{55,56}$ Evaluating ADL performance allows for a more standardized and quantitative assessment of functional limitations. There are two classes of ADLs: 1) basic ADLs, which relate to personal care and mobility (e.g., personal hygiene, dressing, eating, ambulating, and toileting) and 2) instrumental ADLs, which include more complex activities required for a person to interact with their environment (e.g., grocery shopping or banking). ${ }^{55,56}$ There are several indices used to measure ADL performance, such as the Barthel Index and ADL Score (Katz Index). ${ }^{57,58}$

A systematic review of nine studies assessing ADLs (the majority assessing inpatients) found ADL performance declined following SARS-CoV-2 infection, regardless of the scale used. ${ }^{55}$ In a six-month follow-up survey of 177 COVID-19 patients that were asymptomatic, outpatients, or hospitalized, $7.9 \%$ reported negative impacts on at least one $\mathrm{ADL}$, most commonly household chores..$^{59}$ In an NIHR survey, $71 \%$ of respondents said the post COVID-19 condition was affecting their family life, with $39 \%$ saying it was hampering their ability to care for children or other dependents. ${ }^{14}$

An increased presentation of cognitive deficits in individuals who have recovered from COVID-19 has also been noted in a study of cross-sectional cognitive performance data from 81,337 members of the UK general public using an extended questionnaire. ${ }^{28}$ Notably, the standardized assessment of cognitive performance was done prior to individuals self-reporting their COVID-19 status. Individuals with severe COVID-19 (hospitalized and on mechanical ventilation) had a 0.47 standard deviation (SD) composite score reduction compared to controls, equivalent to a 7-point difference in intelligence quotient (IQ). This was greater than the average 10-year decline for individuals in the dataset (age range 20 to 70). It was also larger than the average deficit of 480 people in the sample who had previously suffered a stroke and the 998 who reported learning disabilities.

There are data gaps regarding long-term disability and unemployment for those with the post COVID-19 condition. A study of 150 people with the post COVID-19 condition in France who were treated mainly in outpatient settings found that about $11 \%$ were on sick leave 60 days after the onset of COVID-19 symptoms. Half of this cohort were health care workers, who are typically a younger and healthier group at baseline. ${ }^{60}$ A patient-led study which surveyed 3,762 individuals with suspected or confirmed COVID-19 found that $45.2 \%$ reported requiring a reduced work schedule compared to before illness and $22.3 \%$ were not working at the time of survey due to their health conditions. ${ }^{10}$ These findings are consistent with previous literature tracking outcomes of patients with ARDS which found that $51 \%$ did not return to work. ${ }^{61}$ As well, these findings are similar to findings from the 2003 SARS outbreak in Toronto, in which 22 out of 273 SARS cases (8.1\%) were unable to return to work at least 13 months 
following SARS infection. ${ }^{62}$

The impact of post COVID-19 condition on families and caregivers of patients has not been well researched to date. However, there is evidence that caregivers of patients who have survived critical illness in general experience high levels of persistent depressive symptoms and may experience physical and psychological morbidity. ${ }^{63,64}$ Being a caregiver who is experiencing mental or emotional strain is also an independent risk factor for 4-year mortality. ${ }^{65}$

\section{Expected Burden of Post COVID-19 Condition in Ontario}

Crude estimates of the expected burden of post COVID-19 condition in Ontario can be made by applying a range of prevalence estimates (e.g., UK Office for National Statistics, WHO and PHAC) to data on cumulative SARS-CoV- 2 case numbers for the province of Ontario. ${ }^{1}$ This may underestimate the number of individuals with post COVID-19 condition given a substantial proportion of SARS-CoV-2 cases go unreported. ${ }^{66}$

Applying the prevalence estimate from the UK Office for National Statistics April 2021 report (13.7\%), ${ }^{32}$ approximately 78,000 Ontarians had or are currently experiencing the post COVID-19 condition 12 or more weeks beyond their COVID-19 diagnosis. They used a population-representative sample and asked respondents with a known diagnosis of COVID-19 if they had persistent symptoms (e.g., loss of smell) that were not explained by other causes. These symptoms were found to be 8-fold higher in patients with known COVID-19 diagnosis versus controls, suggesting that symptom prevalence is higher than the general population and specific to COVID-19.

To construct their prevalence estimate for the post COVID-19 condition, the WHO used a rapid evidence assessment with combined estimates from several studies but did not engage in primary data gathering or a systematic review and meta-analyses. Using the WHO prevalence estimate (10\%), approximately 57,000 Ontarians had or are currently experiencing the post COVID-19 condition. $^{31}$

PHAC considered the post COVID-19 condition to be present if an individual had 1 or more symptoms in a list of over 100 reported symptoms (resulting in a prevalence estimate of $56 \%, 95 \% \mathrm{Cl} 34-75 \%)$. This would result in a crude estimate of approximately $318,000(95 \% \mathrm{Cl} 193,000-426,000)$ Ontarians who have or had the post COVID-19 condition. Due to the wide range of symptoms included and lack of controls, the PHAC approach may overestimate the prevalence of the post COVID-19 condition. However, it provides similar estimates to the longest follow-up study to date (conducted in individuals in Wuhan, China), where $49 \%$ of individuals continued to have symptoms at 12 months, and this was at double the rate of age-sex-comorbidity matched controls. ${ }^{34}$

COVID-19 in Ontario has been disproportionately concentrated in populations who are racialized, have lower socioeconomic status, are recent immigrants or essential workers. ${ }^{67}$ Although our estimates are at the level of the province, it is likely that the burden and disability associated with the post COVID-19 condition will also be unequally distributed with greater prevalence in these groups. ${ }^{68}$

\section{Interpretation}

It is likely that an important proportion of individuals with COVID-19 will experience persisting health abnormalities and functional limitations that extend to at least 12 months post infection. The literature on the post COVID-19 condition is still largely in a formative phase and more controlled studies are needed.

Several risk factors for the development of the post COVID-19 condition have been identified in existing literature, though this is still an emerging area of study. Predictive models may represent a promising approach to identify higher-risk groups and 
determine who may benefit from early intervention or surveillance. ${ }^{35}$

The impact of initial treatment and medications on the prevalence, severity, and symptom complex of the post COVID-19 condition is unknown. COVID-19 vaccination reduces the prevalence of the post COVID-19 condition by reducing the chance of infection and through reduced risk of developing the post COVID-19 condition among those with a post-vaccine infection. Research is needed on the impact of currently circulating variants as the majority of current evidence is based on the wild-type (Wuhan) strain of SARS-CoV-2.

Due to the considerable variability in definitions of the condition and study methods, prevalence and potential impact of the post COVID-19 condition are difficult to quantify. Conservative estimates of prevalence of the post COVID-19 condition suggest that there are a substantial number of Ontarians affected. Individuals with the post COVID-19 condition have an array of complex health and supportive needs. Health resource planning, management strategies and relevant government policies will be required to manage the burden of post COVID-19 condition on patients, caregivers, the health care system, and province overall.

\section{Methods Used for This Science Brief}

We searched PubMed, Google Scholar, the COVID-19 Rapid Evidence Reviews, the Joanna Briggs Institute's COVID-19 Special Collection, LitCovid in PubMed, the Oxford COVID-19 Evidence Service, the World Health Organization's Global Literature on Coronavirus Disease, and other COVID-19 specific resources listed by the Guidelines International Network and the McMaster Health Forum. In addition, we retrieved reports citing relevant articles through Google Scholar and reviewed references from identified articles for additional studies.

The COVID-19 Evidence Synthesis Network performed research evidence scans for this Science Brief, published in Evidence Synthesis Briefing Notes. The COVID-19 Evidence Synthesis Network is comprised of organizations in Ontario's evidence synthesis and knowledge translation community who collectively provide high-quality, relevant, and timely synthesized research evidence about COVID-19. The Methods for the evidence scans can be found in the methods sections of the Briefing Notes. The evidence scan titled "Understanding Long COVID-19" was last updated May 7, 2021, ${ }^{12}$ and the evidence scan titled "Effectiveness of Rehabilitation Interventions for People with Long COVID" was last updated on July 10, 2021.

Data for cumulative SARS-CoV-2 case numbers between February 9, 2020 and September 3, 2021 was sourced from the Government of Ontario. ${ }^{70}$

\section{Author Contributions}

FR, PJ, KBB and GMK conceived the Science Brief. FR, GMK and KBB wrote the first draft of the Science Brief. GMK and FR performed the analyses. All authors revised the Science Brief critically for important intellectual content and approved the final version.

\section{References}

1. Government of Ontario. All Ontario: Case numbers and spread. Ontario.ca. Published August 10, 2021. https://covid-19.ontario.ca/data

2. Carfi A, Bernabei R, Landi F, for the Gemelli Against COVID-19 Post-Acute Care Study Group. Persistent symptoms in patients after acute COVID-19. JAMA. 2020;324(6):603-605. https://doi.org/10.1001/jama.2020.12603

3. Yong E. COVID-19 can last for several months. The Atlantic. Published June 4, 2020. https://www.theatlantic.com/health/archive/2020/06/covid-19-coronavirus- 
longterm-symptoms-months/612679/

4. WHO Team. Expanding our understanding of post COVID-19 condition. World Health Organization; 2021:36. https://www.who.int/publications-detailredirect/9789240025035

5. National Institute for Health Care and Excellence. COVID-19 rapid guideline: Managing the long-term effects of COVID-19. NICE. Published December 18, 2020. https://www.nice.org.uk/guidance/ng188/chapter/1-Identifying-peoplewith-ongoing-symptomatic-COVID-19-or-post-COVID-19-syndrome

6. CDC. Post-COVID conditions. Centers for Disease Control and Prevention. Published July 12, 2021. https://www.cdc.gov/coronavirus/2019-ncov/long-termeffects.html

7. Domingo FR, Waddell LA, Cheung AM, et al. Prevalence of long-term effects in individuals diagnosed with COVID-19: A living systematic review. medRxiv. Published online June 6, 2021:2021.06.03.21258317. https://doi. org/10.1101/2021.06.03.21258317

8. Azoulay E, Vincent J-L, Angus DC, et al. Recovery after critical illness: Putting the puzzle together-a consensus of 29. Crit Care Lond Engl. 2017;21(1):296. https:// doi.org/10.1186/s13054-017-1887-7

9. Herridge MS, Chu LM, Matte A, et al. The RECOVER program: Disability risk groups and 1-year outcome after 7 or more days of mechanical ventilation. Am J Respir Crit Care Med. 2016;194(7):831-844. https://doi.org/10.1164/rccm.201512$23430 \mathrm{C}$

10. Davis HE, Assaf GS, McCorkell L, et al. Characterizing long COVID in an international cohort: 7 months of symptoms and their impact. EClinicalMedicine. Published online July 15, 2021. https://doi.org/10.1016/j.eclinm.2021.101019

11. Crook H, Raza S, Nowell J, Young M, Edison P. Long covid-mechanisms, risk factors, and management. BMJ. 2021;374:n1648. https://doi.org/10.1136/bmj. n1648

12. Research, Analysis, and Evaluation Branch. Understanding Long COVID-19. COVID-19 Evidence Synthesis Network; 2021. https://esnetwork.ca/wp-content/ uploads/2021/04/Evidence-Synthesis-BN_Understanding-Long-COVID_May-152021_v2.pdf

13. Nasserie T, Hittle M, Goodman SN. Assessment of the frequency and variety of persistent symptoms among patients with COVID-19: A systematic review. JAMA Netw Open. 2021;4(5):e2111417-e2111417. https://doi.org/10.1001/ jamanetworkopen.2021.11417

14. Maxwell E, Poole R. Living with Covid19 - second review. NIHR. Published online March 16, 2021:36. https://doi.org/10.3310/themedreview_45225

15. Poenaru S, Abdallah SJ, Corrales-Medina V, Cowan J. COVID-19 and postinfectious myalgic encephalomyelitis/chronic fatigue syndrome: A narrative review. Ther Adv Infect Dis. 2021;8:20499361211009384. https://doi. org/10.1177/20499361211009385

16. Poole-Wright K, Gaughran F, Evans R, Chalder T. Fatigue outcomes following coronavirus or influenza virus infection: A systematic review and meta-analysis. medRxiv. Published online December 9, 2020:2020.12.04.20244145. https://doi. org/10.1101/2020.12.04.20244145

17. Lam MH-B, Wing Y-K, Yu MW-M, et al. Mental morbidities and chronic fatigue in Severe Acute Respiratory Syndrome survivors: Long-term follow-up. Arch Intern 
Med.2009;169(22):2142-2147. https://doi.org/10.1001/archinternmed.2009.384

18. The RECOVERY Collaborative Group. Dexamethasone in hospitalized patients with Covid-19. N Engl J Med. 2021;384(8):693-704. https://doi.org/10.1056/ NEJMoa2021436

19. Abani $O$, Abbas $A$, Abbas $F$, et al. Tocilizumab in patients admitted to hospital with COVID-19 (RECOVERY): A randomised, controlled, open-label, platform trial. The Lancet. 2021;397(10285):1637-1645. https://doi.org/10.1016/S01406736(21)00676-0

20. Long-term immunological health consequences of COVID-19. British Society for Immunology;2020:13. https://www.immunology.org/sites/default/files/BSI_ Briefing_Note_August_2020_FINAL.pdf

21. Raman B, Cassar MP, Tunnicliffe EM, et al. Medium-term effects of SARS-CoV-2 infection on multiple vital organs, exercise capacity, cognition, quality of life and mental health, post-hospital discharge. EClinicalMedicine. 2021;31. https://doi. org/10.1016/j.eclinm.2020.100683

22. Mo X, Jian W, Su Z, et al. Abnormal pulmonary function in COVID-19 patients at time of hospital discharge. Eur Respir J. Published online January 1, 2020. https:// doi.org/10.1183/13993003.01217-2020

23. Mandal S, Barnett J, Brill SE, et al. 'Long-COVID': A cross-sectional study of persisting symptoms, biomarker and imaging abnormalities following hospitalisation for COVID-19. Thorax. 2021;76(4):396-398. https://doi.org/10.1136/ thoraxjnl-2020-215818

24. Cui S, Chen S, Li X, Liu S, Wang F. Prevalence of venous thromboembolism in patients with severe novel coronavirus pneumonia. J Thromb Haemost JTH. 2020;18(6):1421-1424. https://doi.org/10.1111/jth.14830

25. Puntmann VO, Carerj ML, Wieters I, et al. Outcomes of cardiovascular magnetic resonance imaging in patients recently recovered from Coronavirus disease 2019 (COVID-19). JAMA Cardiol. 2020;5(11):1265-1273. https://doi.org/10.1001/ jamacardio.2020.3557

26. Bowe B, Xie Y, Xu E, Al-Aly Z. Kidney outcomes in long COVID. J Am Soc Nephrol. Published online August 31, 2021. https://doi.org/10.1681/ASN.2021060734

27. Ayoubkhani D, Khunti K, Nafilyan V, et al. Post-covid syndrome in individuals admitted to hospital with covid-19: Retrospective cohort study. BMJ. 2021;372:n693. https://doi.org/10.1136/bmj.n693

28. Hampshire A, Trender W, Chamberlain SR, et al. Cognitive deficits in people who have recovered from COVID-19. EClinicalMedicine. 2021;0(0). https://doi. org/10.1016/j.eclinm.2021.101044

29. Sudre $\mathrm{CH}$, Murray B, Varsavsky T, et al. Attributes and predictors of long-COVID: Analysis of COVID cases and their symptoms collected by the Covid symptoms study app.; 2020:2020.10.19.20214494. https://doi.org/10.1101/2020.10.19.20214494

30. D'Cruz RF, Waller MD, Perrin F, et al. Chest radiography is a poor predictor of respiratory symptoms and functional impairment in survivors of severe COVID-19 pneumonia. ERJ Open Res. 2021;7(1):00655-02020. https://doi. org/10.1183/23120541.00655-2020

31. Rajan S, Khunti K, Alwan NA, et al. In the wake of the pandemic: Preparing for Long COVID (2021). Eur Obs Health Syst Policies. (39):30. https://www.euro. who.int/en/about-us/partners/observatory-old/publications/policy-briefs-andsummaries/in-the-wake-of-the-pandemic-preparing-for-long-covid-2021 
32. Ayoubkhani D. Prevalence of ongoing symptoms following coronavirus (COVID-19) infection in the UK: 1 April 2021. Office for National Statistics. Published April 1, 2021. https://www.ons.gov.uk/peoplepopulationandcommunity/ healthandsocialcare/conditionsanddiseases/bulletins/ prevalenceofongoingsymptomsfollowingcoronaviruscovid19infectionintheuk/

33. Huang C, Huang L, Wang Y, et al. 6-month consequences of COVID-19 in patients discharged from hospital: A cohort study. The Lancet. 2021;397(10270):220-232. https://doi.org/10.1016/S0140-6736(20)32656-8

34. Huang L, Yao Q, Gu X, et al. 1-year outcomes in hospital survivors with COVID-19: A longitudinal cohort study. The Lancet. 2021;398(10302):747-758. https://doi. org/10.1016/S0140-6736(21)01755-4

35. Sudre $\mathrm{CH}$, Murray B, Varsavsky T, et al. Attributes and predictors of long COVID. Nat Med. 2021;27(4):626-631. https://doi.org/10.1038/s41591-021-01292-y

36. Kamal M, Abo Omirah M, Hussein A, Saeed H. Assessment and characterisation of post-COVID-19 manifestations. Int J Clin Pract. 2021;75(3):e13746. https://doi. org/10.1111/ijcp.13746

37. Ludvigsson JF. Case report and systematic review suggest that children may experience similar long-term effects to adults after clinical COVID-19. Acta Paediatr. 2020;110(3):914-921. https://doi.org/10.1111/apa.15673

38. Tenforde MW, Kim SS, Lindsell CJ, et al. Symptom duration and risk factors for delayed return to usual health among outpatients with COVID-19 in a multistate health care systems network - United States, March-June 2020. MMWR - Morb Mortal Wkly Rep. 2020;69(30):993-998. https://doi.org/10.15585/mmwr. mm6930e1

39. Nabavi N. Long covid: How to define it and how to manage it. BMJ. 2020;370. https://doi.org/10.1136/bmj.m3489

40. Bergwerk $M$, Gonen $T$, Lustig $Y$, et al. Covid-19 breakthrough infections in vaccinated health care workers. N Engl J Med. Published online July 28, 2021. https://doi.org/10.1056/NEJMoa2109072

41. Antonelli M, Penfold RS, Merino J, et al. Risk factors and disease profile of postvaccination SARS-CoV-2 infection in UK users of the COVID symptom study app: A prospective, community-based, nested, case-control study. Lancet Infect Dis. Published online September 1, 2021. https://doi.org/10.1016/S14733099(21)00460-6

42. Rochon PA, Wu W, Giannakeas V, Stall NM. The missing pieces of the COVID-19 puzzle. J Am Geriatr Soc. 2020;68(8):1671-1673. https://doi.org/10.1111/ jgs.16646

43. Akerman JM, Rochon PA. The epidemiology of COVID-19: A focus on sex, gender and age. Ital J Gend-Specif Med. 2021;7(3). Accessed September 8, 2021. https://www. gendermedjournal.it/r.php?v=3597\&a=36246\&l=345336\&f=allegati/00000_00/ fulltext/36246_-Abstract\%20from\%20IGM_1_Rochon.pdf

44. Irfan O, Muttalib F, Tang K, Jiang L, Lassi ZS, Bhutta Z. Clinical characteristics, treatment and outcomes of paediatric COVID-19: A systematic review and meta-analysis. Arch Dis Child. 2021;106(5):440-448. https://doi.org/10.1136/ archdischild-2020-321385

45. Molteni E, Sudre $\mathrm{CH}$, Canas LS, et al. Illness duration and symptom profile in symptomatic UK school-aged children tested for SARS-CoV-2. Lancet Child Adolesc Health. 2021;0(0). https://doi.org/10.1016/S2352-4642(21)00198-X 
46. Dougherty K. SARS-CoV-2 B.1.617.2 (Delta) variant COVID-19 outbreak associated with a gymnastics facility - Oklahoma, April-May 2021. MMWR Morb Mortal Wkly Rep. 2021;70. https://doi.org/10.15585/mmwr.mm7028e2

47. Menges D, Ballouz T, Anagnostopoulos A, et al. Burden of post-COVID-19 syndrome and implications for healthcare service planning: A population-based cohort study. PLOS ONE. 2021;16(7):e0254523. https://doi.org/10.1371/journal.pone.0254523

48. Lavery AM, Preston LE, Ko JY, et al. Characteristics of hospitalized COVID-19 patients discharged and experiencing same-hospital readmission - United States, March-August 2020. MMWR Morb Mortal Wkly Rep. 2020;69(45):1695-1699. https://doi.org/10.15585/mmwr.mm6945e2

49. Loerinc LB, Scheel AM, Evans ST, Shabto JM, O'Keefe GA, O'Keefe JB. Discharge characteristics and care transitions of hospitalized patients with COVID-19. Healthc Amst Neth. 2020;9(1). https://doi.org/10.1016/j.hjdsi.2020.100512

50. Chopra V, Flanders SA, O'Malley M, Malani AN, Prescott HC. Sixty-day outcomes among patients hospitalized with COVID-19. Ann Intern Med. 2021;174(4):576578. https://doi.org/10.7326/M20-5661

51. Donnelly JP, Wang XQ, Iwashyna TJ, Prescott HC. Readmission and death after initial hospital discharge among patients with COVID-19 in a large multihospital system. JAMA. 2021;325(3):304-306. https://doi.org/10.1001/jama.2020.21465

52. Clark JR, Batra A, Shlobin NA, et al. Acute-care hospital reencounters in COVID-19 patients. GeroScience. Published online May 21, 2021:1-13. https://doi. org/10.1007/s11357-021-00378-2

53. Chua K-P, Conti RM, Becker NV. Out-of-Pocket Spending Within 90 Days of Discharge from COVID-19 Hospitalization. medRxiv. Published online June 18, 2021:2021.06.11.21258766. https://doi.org/10.1101/2021.06.11.21258766

54. Hernandez-Romieu AC. Health care utilization and clinical characteristics of non-hospitalized adults in an integrated health care system 28-180 days after COVID-19 diagnosis - Georgia, May 2020-March 2021. Morb Mortal Wkly Rep MMWR. 2021;70:664-650. https://doi.org/10.15585/mmwr.mm7017e3

55. Pizarro-Pennarolli C, Sánchez-Rojas C, Torres-Castro R, et al. Assessment of activities of daily living in patients post COVID-19: A systematic review. PeerJ. 2021;9:47. https://doi.org/10.7717/peerj.11026

56. Edemekong PF, Bomgaars DL, Sukumaran S, Levy SB. Activities of daily living. StatPearls Publishing; 2021. https://www.ncbi.nlm.nih.gov/books/NBK470404/

57. Barthel index of activities of daily living. Published online April 2015. https:// www.albertahealthservices.ca/assets/about/scn/ahs-scn-bjh-hf-barthel-index-ofadls.pdf

58. Shelkey M, Wallace M. Katz Index of independence in activities of daily living. J Gerontol Nurs. 1999;25(3):8-9. https://doi.org/10.3928/0098-9134-19990301-05

59. Logue JK, Franko NM, McCulloch DJ, et al. Sequelae in adults at 6 months after COVID-19 infection. JAMA Netw Open. 2021;4(2):e210830-e210830. https://doi. org/10.1001/jamanetworkopen.2021.0830

60. Yelin D, Margalit I, Yahav D, Runold M, Bruchfeld J. Long COVID-19-it's not over until? Clin Microbiol Infect. 2021;27(4):506-508. https://doi.org/10.1016/j. cmi.2020.12.001

61. Herridge MS, Cheung AM, Tansey CM, et al. One-year outcomes in survivors of the acute respiratory distress syndrome. N Engl J Med. 2003;348(8):683-693. https:// doi.org/10.1056/NEJMoa022450 
62. Moldofsky H, Patcai J. Chronic widespread musculoskeletal pain, fatigue, depression and disordered sleep in chronic post-SARS syndrome; a case-controlled study. BMC Neurol. 2011;11(1):37. https://doi.org/10.1186/1471-2377-11-37

63. Cameron JI, Chu LM, Matte A, et al. One-year outcomes in caregivers of critically ill patients. N Engl J Med. 2016;374(19):1831-1841. https://doi.org/10.1056/ NEJMoa1511160

64. Dale CM, Carbone S, Istanboulian L, et al. Support needs and health-related quality of life of family caregivers of patients requiring prolonged mechanical ventilation and admission to a specialised weaning centre: A qualitative longitudinal interview study. Intensive Crit Care Nurs. 2020;58:102808. https://doi.org/10.1016/j. iccn.2020.102808

65. Schulz R, Beach SR. Caregiving as a risk factor for mortality: The caregiver health effects study. JAMA. 1999;282(23):2215-2219. https://doi.org/10.1001/ jama.282.23.2215

66. COVID-19 in Ontario: January 15, 2020 to August 25, 2021. Public Health Ontario; 2021:27. https://www.publichealthontario.ca/-/media/documents/ncov/ epi/2020/covid-19-daily-epi-summary-report.pdf?la=en

67. Mishra S, Ma H, Moloney G, et al. Increasing concentration of COVID-19 by socioeconomic determinants and geography in Toronto, Canada: An observational study. medRxiv. Published online April 6, 2021:2021.04.01.21254585. https://doi. org/10.1101/2021.04.01.21254585

68. Briggs A, Vassall A. Count the cost of disability caused by COVID-19. Nature. 2021;593(7860):502-505. https://doi.org/10.1038/d41586-021-01392-2

69. Research, Analysis, and Evaluation Branch. Effectiveness of rehabilitation interventions for people with long COVID. COVID-19 Evidence Synthesis Network; 2021:14. https://esnetwork.ca/wp-content/uploads/2021/07/63.-EvidenceSynthesis-BN-on-Benefits-of-Long-COVID-19-Rehabilitation_15-July-2021.pdf

70. Government of Ontario. Status of COVID-19 cases in Ontario. Published 2020. https://data.ontario.ca/dataset/status-of-covid-19-cases-in-ontario 